\title{
CARACTERIZAÇÃO ESPECTRAL NA REFLECTÂNCIA DE Eucalyptus grandis
}

\author{
Emanuel Araújo Silva ${ }^{1}$, Gabriel Paes Marangon ${ }^{1}$, Luana Dessbesell ${ }^{2}$, Weslley Wilker Morais ${ }^{3}$, \\ Diogo Belmonte Lippert ${ }^{1}$, Rudiney Soares Pereira ${ }^{4}$
${ }^{1}$ Eng. Florestal, Doutorando em Engenharia Florestal, UFSM, Santa Maria, RS, Brasil - mad_emanuel@hotmail.com; gabrimarangon@yahoo.com.br; diogo_b_lippert@hotmail.com
${ }^{2}$ Acadêmica em Engenharia Florestal, UFSM, Santa Maria, RS, Brasil - luana.dessbesell@ gmail.com
${ }^{3}$ Eng. Florestal, M.Sc., UFSM, Santa Maria, RS, Brasil - weslley_eng@ hotmail.com \\ ${ }^{4}$ Eng. Florestal, Dr., Depto. de Engenharia Rural, UFSM, Santa Maria, RS, Brasil - rudiney.s.pereira@ gmail.com
}

Recebido para publicação: 27/05/2010 - Aceito para publicação: 26/03/2012

\begin{abstract}
Resumo
O objetivo do trabalho foi comparar níveis de reflectância das folhas de Eucalyptus grandis Hill ex Maiden através de medidas espectrais a partir da localização das mesmas em relação à árvore e aos pontos cardeais. O experimento foi conduzido na área experimental pertencente à FEPAGRO, localizada no município de Santa Maria-RS. O trabalho compreendeu a instalação de delineamento blocos ao acaso. Com a utilização de um espectrorradiômetro FieldSpec $® 3$, foram obtidas as medidas de radiância refletida da face superior das folhas e posteriormente trabalhados no software R. Na Banda 1 (400 a $700 \mathrm{~nm}$ ), notou-se um pico que está relacionado ao total de clorofila na folha dentro da faixa do visível. Observou-se que folhas na posição leste foram as que apresentaram maior reflectância, e as folhas que estavam ao sul e ao oeste apresentaram as menores. Para a Banda 2 (700 a $1000 \mathrm{~nm})$, observou-se diferença estatística significativa apenas entre os valores médios das reflectâncias no par de posições leste-oeste, contrastando com a Banda 1, que obteve diferença significativa entre as reflectâncias médias de todos os pares. A resposta espectral das folhas revelou-se estatisticamente diferente, confirmando, assim, que há diferença espectral relacionada à localização das árvores e posições de coletas das folhas.

Palavras-chave: Espectrorradiometria; sensoriamento remoto; curva de reflectância.
\end{abstract}

\begin{abstract}
Spectral reflectance characterization in Eucalyptus grandis. The objective was to compare levels of reflectance of leaves of Eucalyptus grandis Hill ex Maiden using spectral measurements from their location in relation to the tree and the cardinal points. The experiment was carried out in the experimental area belonging to FEPAGRO, located in Santa Maria-RS. The work included the installation of a randomized block design. With the use of a spectroradiometer FieldSpec ${ }^{\circledR}$ 3, it was measured the radiance reflected from the upper surface of leaves for further work with the software R. In the first band (400 to $700 \mathrm{~nm}$ ), it was realized that a peak is related to total chlorophyll within the visible range. It was observed that the leaves heading east presented the highest reflectance and the leaves at south and west had the lowest one. For the second band (700 to $1000 \mathrm{~nm}$ ), we observed a statistically significant difference only between the average values of reflectance in the pair of east-west positions, contrasting with the band that got a significant difference between the average reflectance of all pairs. The spectral response of leaves proved to be statistically different; it confirms that there are differences related to the spectral location of trees and locations of collected leaves.
\end{abstract}

Keywords: Spectroradiometry; remote sensing; reflectance curve.

\section{INTRODUÇÃO}

Técnicas ligadas ao sensoriamento remoto têm sido utilizadas para análise do comportamento da vegetação em diferentes paisagens, entre elas, floresta, agricultura e vegetação urbana. Estudos vêm sendo realizados abordando a resposta de objetos à radiação, sendo o solo e a vegetação os elementos mais pesquisados na área de ciências agrárias. 
O conhecimento da interação entre a vegetação e o meio que a cerca tem grande importância para o entendimento dos fenômenos naturais. A espectrorradiometria revela-se uma ferramenta que possibilita detectar a resposta espectral através do contato direto com o alvo e pode ser feita em laboratório, permitindo assim que se minimizem as interferências dos fatores ambientais presentes nas leituras de outros sensores. A partir de dados obtidos com a utilização dessa ferramenta, é possível obter diversas informações referentes à forma como a vegetação processa a radiação eletromagnética e de como se dá na distribuição dos diferentes tipos de vegetação, no estado fenológico, na estrutura do dossel, na carência de nutrientes e nas condições de estresse, entre outros fatores.

De acordo com Gates et al. (1965), três mecanismos influenciam a quantidade de energia eletromagnética refletida pelas folhas: pigmentos, espaços celulares ocupados pela água e pelo ar e estruturas celulares com dimensões do comprimento de onda da radiação incidente (grãos de amido, mitocôndrias, ribossomos, núcleo e outros plastídios). Para Gausman (1985), além desses três mecanismos, outros fatores afetam a energia refletida pelas folhas: conteúdo de água, maturação ou idade da folha, posição nodal, condição de iluminação (folhas expostas ao sol e folhas constantemente à sombra), pubescência e senescência. Datt (1998) complementa que, em uma folha, a reflexão e a absorção da luz são influenciadas por dois processos: dispersão de luz como resultado da superfície de uma folha e estrutura celular interna, e absorção da energia radiante ditada pela bioquímica foliar.

A figura 1 proposta por Jensen (2009) apresenta o espectro de reflectância de uma folha verde sadia. Nela podemos observar e confirmar a relação de características como pigmentos, espaços celulares e água foliar na reflectância de uma folha verde e sadia.

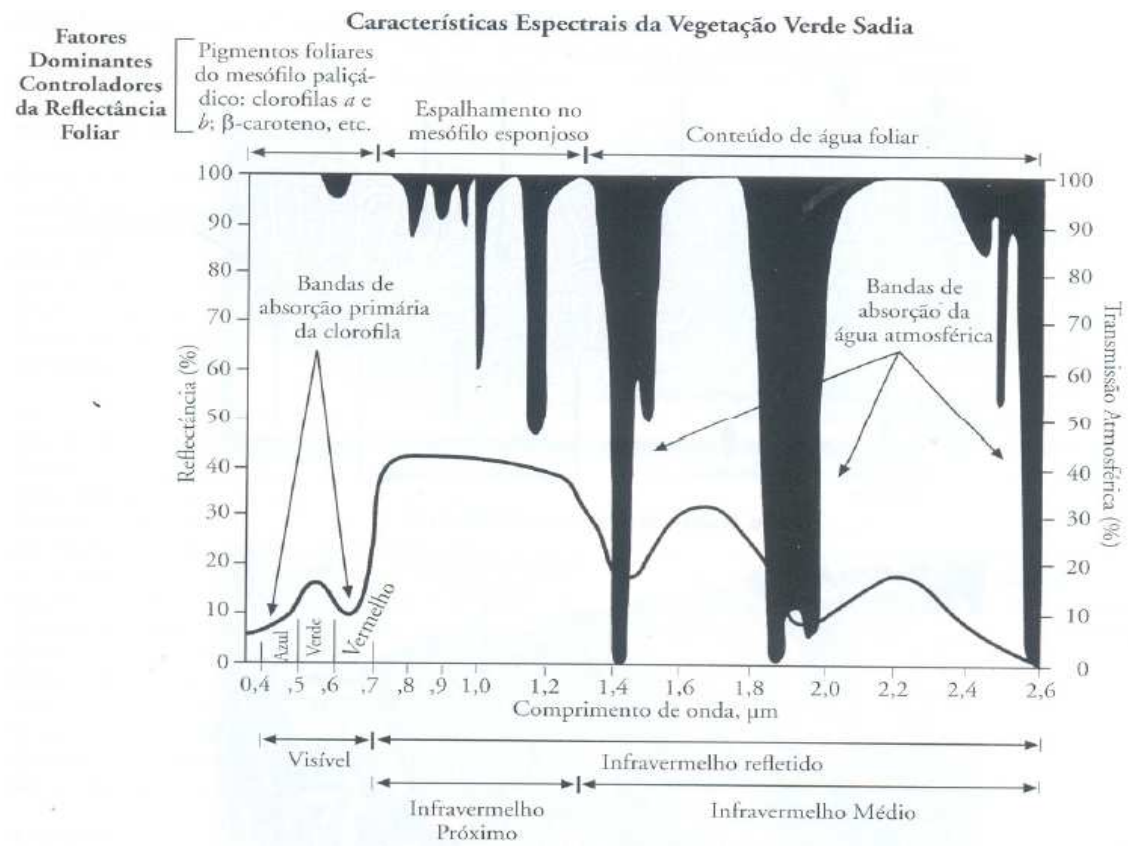

Figura 1. Espectro de reflectância de uma folha verde e sadia.

Figure 1. Reflectance spectrum of a healthy green leaf.

As publicações internacionais demonstram a evolução dos estudos de forma exponencial, com destaque para vários autores (Curran et al., 1990; Peñuelas e Filella, 1998; Trigg e Flasse, 2000; entre outros). No Brasil também vários pesquisadores buscaram compreender a relação da reflectância com comportamento de alvos terrestres: Moreira (1997) analisou o comportamento do trigo, Brandeleiro (2010) estudou Eucalyptus grandis e Epiphanio et al. (1992) avaliaram o comportamento espectral de solos no estado de São Paulo.

Mesmo com o advento de trabalhos nessa área, ainda têm-se um vasto campo a ser estudado no que diz respeito a espécies florestais nativas e exóticas. Entende-se que, com a aplicação do conhecimento dos fatores fisiológicos influentes na reflectância, relacionando-os aos fatores ambientais, possamos criar 
subsídios que nos auxiliem no entendimento da reflectância das espécies florestais de importância social e econômica.

No Brasil, no âmbito econômico, podemos destacar o gênero Eucalyptus como o mais importante no que diz respeito a florestas plantadas. Existem no país mais de 3 milhões de ha de plantações desse gênero, sendo as espécies mais cultivadas Eucalyptus grandis, Eucalyptus citriodora, Eucalyptus camaldulensis, Eucalyptus saligna e Eucalyptus urophilla (ANUÁRIO ABRAF, 2010).

A espécie Eucalyptus grandis tem grande importância econômica, devido a sua vasta abrangência de usos, como, por exemplo, celulose, lenha, caixotaria e laminação. Além disso, essa espécie se adapta à maioria dos solos e tem considerável resistência a climas frios e úmidos.

Diante do exposto, pretende-se avaliar diferentes níveis de reflectância de folhas extraídas de Eucalyptus grandis Hill ex Maiden utilizando-se medidas espectrais em diferentes exposições na árvore em relação aos pontos cardeais. Inferir o melhor intervalo para diferenciá-las e enfatizar a importância do estudo da variação natural da curva de reflectância para espécies florestais.

\section{MATERIAIS E MÉTODOS}

\section{Área do estudo}

$\mathrm{O}$ experimento foi conduzido na área experimental pertencente à Fundação Estadual de Pesquisa Agropecuária, Centro de Pesquisas de Recursos Florestais (FEPAGRO FLORESTAS), localizada no município de Santa Maria, região fisiográfica da depressão central do estado do Rio Grande do Sul, localizada entre as coordenadas geográficas $29^{\circ} 40^{\prime} 31^{\prime \prime}$ de latitude Sul e 53 $54^{\prime} 45^{\prime \prime}$ de longitude Oeste de Greenwich, com altitude média de 130 metros.

O clima da região, segundo a classificação de Köppen, é do tipo Cfa, subtropical úmido, sem estiagens. Tem como características climáticas principais a temperatura média anual de $19^{\circ} \mathrm{C}$, precipitação média anual de $1.769 \mathrm{~mm}$ e umidade relativa de 82\% (MORENO, 1961). A vegetação natural predominante é a de campo. As matas nativas são representadas apenas por formações de galerias ao longo de cursos d'água (ABRÃO et al., 1988).

O solo estudado é classificado como Argissolo Vermelho-Amarelo Distrófico arênico (EMPRESA BRASILEIRA DE PESQUISA AGROPECUÁRIA - EMBRAPA, 1999), pertencente à Unidade de Mapeamento São Pedro (BRASIL, 1973). Essa unidade de mapeamento caracteriza-se por apresentar solos mediamente profundos, não hidromórficos, avermelhados, textura superficial franco arenosa, friáveis e bem drenados, possuindo B textural. São solos ácidos e pobres em matéria orgânica (STRECK et al., 2002).

\section{Descrição do povoamento}

O povoamento pertence à espécie Eucalyptus grandis Hill ex Maiden, oriundo de sementes do pomar clonal da Rigesa, e foi implantado em novembro de 2006 sob área total de 0,72 ha, espaçamento $3 \times 2 \mathrm{~m}$ em diferentes preparos de solo na linha de plantio. Como preparo, tem-se: plantio direto, escarificação, grade niveladora e enxada rotativa. Antes da instalação das parcelas, foi realizada roçada mecânica em toda a área e o controle de formigas (PREVEDELLO, 2008).

\section{Obtenção de dados em campo}

No dia 08 de outubro de 2009, por volta das 15:00 h, para a realização do experimento, foram coletados um total de 2400 amostras de folhas de E. grandis. Elas ficavam na porção inferior da copa da árvore e foram divididas conforme a localização dos quatro pontos cardeais. As folhas obtidas foram armazenadas em embalagens plásticas identificadas e acondicionadas em caixas térmicas sob temperatura média de $15^{\circ} \mathrm{C}$ para posterior análise em laboratório.

O experimento compreendeu a instalação de Delineamento Blocos ao Acaso (DBC), representado por três blocos com quatro parcelas de $600 \mathrm{~m}^{2}$ cada, totalizando a área do bloco em 2.400 $\mathrm{m}^{2}$. Das 100 árvores presentes em cada parcela, foram selecionadas cinco árvores médias, as quais representariam a parcela, e de cada uma delas coletaram-se 10 folhas em cada posição - norte, sul, leste e oeste (Figura 2).

\section{Medições radiométricas}

As medições radiométricas foram realizadas com aproximadamente 30 minutos de coleta, evitando dessa forma a deterioração e alteração das condições naturais do material vegetal. Os 
procedimentos ocorreram no Laboratório de Sensoriamento Remoto da UFSM (LABSERE), com a utilização de um espectrorradiômetro FieldSpec ${ }^{\circledR} 3$ conectado à unidade RTS-3ZC 3 (esfera integradora). Foram obtidas então as medidas de radiância refletida da face superior das folhas para todas as posições de coleta. Um microcomputador acoplado ao espectrorradiômetro armazenou as medidas radiométricas à medida que foram sendo obtidas. Os dados originais foram convertidos para o formato texto com o uso do software ASD ViewSpecPro Versão 4.05, para posterior processamento estatístico no software R.

A

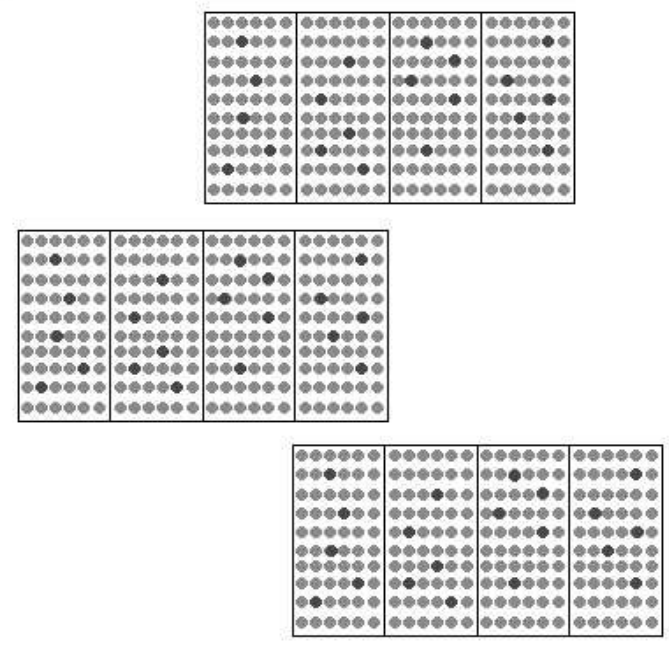

B

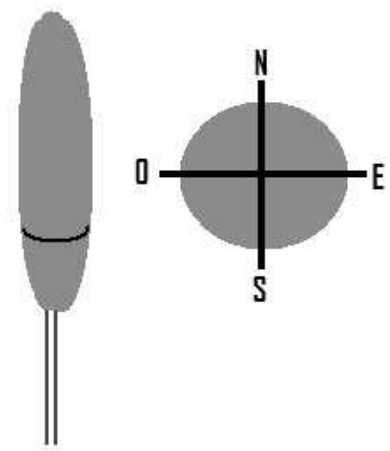

Figura 2. A: exemplo da distribuição dos blocos e parcelas, e em preto as árvores médias; B: posição da coleta do material vegetativo, parte inferior da copa e nas posições norte, sul, leste e oeste.

Figure 2. A: example of distribution of blocks and plots, in black the medium trees; B: position of the material collected, the bottom of the cup and the views north, south, east and west.

O espectrorradiômetro FieldSpec possui capacidade de registro de reflectâncias que vão do comprimento de onda de 300 a $2500 \mathrm{\eta m}$, no entanto foram selecionados, para a análise estatística, os valores correspondentes à faixa do visível, compreendidos no intervalo espectral de 400 a 700 ๆm, e do infravermelho próximo do espectro eletromagnético, compreendidos no intervalo entre 700 e $1.100 \eta \mathrm{m}$. Essa subdivisão é proposta em estudos de reflectância de alvos como a vegetação por autores como Ponzoni e Shimabukuro (2010), Ponzoni (2001) e Jensen et al. (2009).

\section{Procedimento estatístico}

Uma vez dispostos na planilha, foi determinado o fator de reflectância médio para cada posição. Para análise dos resultados, foi adotado o software "R: A Programming Environment for Data Analysis and Graphics", versão 2.8.0, 2008. Os resultados de reflectância foram dispostos em delineamento inteiramente casualizado por não haver diferença entre os blocos, e em função de não terem sido significativos os preparos de solo. Somente as posições de coleta das folhas em relação aos pontos cardeais apresentaram significância e foram consideradas fatores de variação, os quais podem ser observados nas tabelas de ANOVA presente nos resultados. Posteriormente as médias foram submetidas ao teste de comparação múltipla de médias de Tukey (função Tukey HSD) a 5\% de significância.

\section{RESULTADOS E DISCUSSÃO}

\section{Interpretação gráfica}

A leitura do material foi realizada logo após a sua coleta, apresentando aspecto visual de cor verde intensa e escura, não distinguindo de sua condição natural. Na figura 3 observa-se que a reflectância segue a mesma tendência para os diferentes tratamentos. Na região próxima dos $550 \mathrm{~nm}$, nota-se um pico que está relacionado ao total de clorofila na folha dentro da faixa do visível (SCHEPERS 
et al., 1996). Nessa região, também pode ser observado que folhas na posição leste foram as que apresentaram maior reflectância e que as folhas que estavam ao sul e ao oeste apresentaram as menores reflectâncias.



Figura 3. Reflectância x comprimento de onda (400 a $700 \mathrm{~nm})$.

Figure 3. Reflectance $x$ wavelength (400 to $700 \mathrm{~nm}$ ).

Considerando que, na tomada da leitura, as folhas ainda realizavam fotossíntese, a curva espectral resultante têm características de vegetação sadia, com bandas de absorção devidas à clorofila nas regiões do azul e do vermelho (próximo de $5 \%$ de reflectância em $450 \mathrm{~nm}$ e $650 \mathrm{~nm}$ ), além de um pico de reflectância no verde (aproximadamente $8,5 \%$ em $550 \mathrm{~nm}$ ).

Estudos com folhas de Liquidanbar styraciflua L., realizados por Jensen (2009), constataram que para as folhas verdes sadias a reflectância observada foi de $6 \%$ no comprimento de onda $450 \mathrm{~nm}$ e $5 \%$ em $650 \mathrm{~mm}$, abrangendo assim a região do azul e do vermelho, além de um pico no verde de $11 \% \mathrm{em}$ $550 \mathrm{~nm}$. Esse valores são semelhantes aos encontrados para o Eucalyptus grandis neste trabalho.

A figura 4 demonstra as curvas de reflectância da espécie no intervalo de 700 a $1000 \mathrm{~nm}$. Na análise visual pode-se constatar que há uma inclinação na região de $750 \mathrm{~nm}$ e que a partir desse ponto a curva estabiliza.

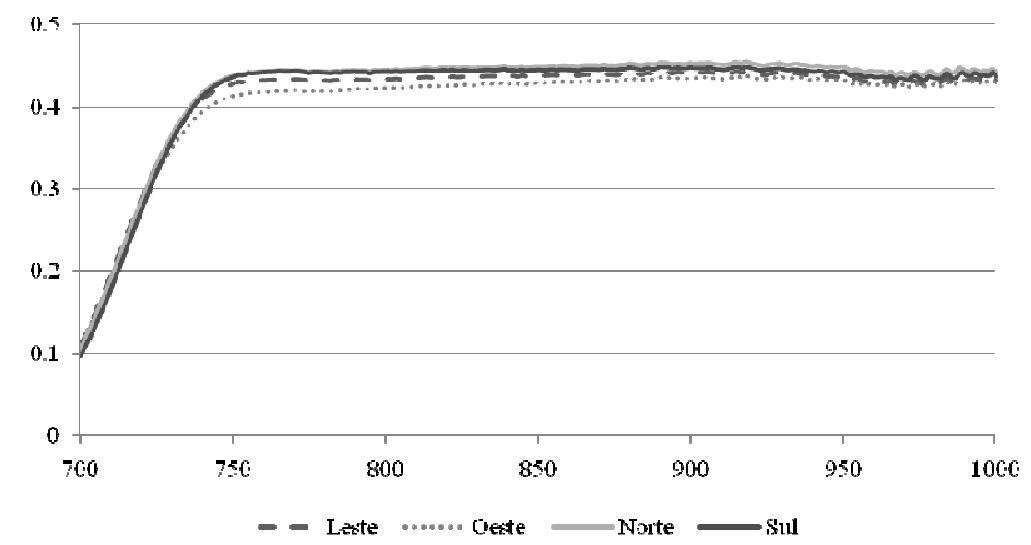

Figura 4. Reflectância x comprimento de onda (700 a $1000 \mathrm{~nm})$.

Figure 4. Reflectance $\mathrm{x}$ wavelength (700 to $1000 \mathrm{~nm}$ ). 
A inclinação, de acordo com Thenkabail et al. (2000), indica o ponto crítico em torno do limite do espectro vermelho. Nessa região, a refletância é sensível ao estresse da vegetação e fornece a informação adicional sobre o status de clorofila e do nitrogênio das plantas (CLEVERS, 1989). Na região do infravermelho próximo $(\mathrm{em} 800 \mathrm{~nm}$ ), aproximadamente $45 \%$ do fluxo radiante incidente foram refletidos pela folha. Na figura 1, apresentada na revisão bibliográfica, podemos observar a semelhança com as curvas das figuras 3 e 4 .

\section{Análise estatística}

As diferenças estatísticas foram significativas entre os tratamentos representados na análise de variância (ANOVA) (Tabela 1). O teste de Tukey HSD (Tabela 2) foi realizado para observar os contrastes entre as médias (duas a duas), ou seja, em quais pares houve maior diferença das posições no povoamento, e verificar quais médias foram significativamente diferentes.

Tabela 1. ANOVA para Banda $1(400$ a $700 \mathrm{~nm})$, para as orientações norte, sul, leste e oeste do povoamento.

Table 1. ANOVA in Band 1 (400 to $700 \mathrm{~nm})$, for guidelines North, South, East and West of the population.

\begin{tabular}{lccccc}
\hline Fator de variação & GL & SQ & QM & P valor calc. & P valor tab. \\
\hline Posição (árvore) & 3 & 0,03459 & 0,01153 & 36,832 & $2,20 \mathrm{E}-16^{* * * *}$ \\
Erro & 1200 & 0,375655 & 0,000313 & & \\
\hline
\end{tabular}

Tabela 2. Interações ocorridas entre as posições na Banda 1 (400 a $700 \mathrm{~nm})$.

Table 2. Interactions between positions in the Band 1 (400 to $700 \mathrm{~nm})$.

\begin{tabular}{lcccc}
\hline Posições & $\begin{array}{c}\text { Diferenças } \\
\text { (diff) }\end{array}$ & $\begin{array}{c}\text { Limite inferior } \\
\text { (lwr) }\end{array}$ & $\begin{array}{c}\text { Limite superior } \\
\text { (upr) }\end{array}$ & p adj \\
\hline Norte-Sul & 0,004358 & 0,000647394 & 0,008068 & 0,013673 \\
Oeste-Sul & 0,013914 & 0,010203302 & 0,017624 & 0 \\
Norte-Leste & 0,012085 & 0,008374235 & 0,015795 & 0 \\
Oeste-Norte & 0,009556 & 0,005845591 & 0,013266 & 0 \\
\hline
\end{tabular}

Na tabela 2, os pares (Posições) com diferenças significativas são aqueles com limites inferiores (lwr) positivos. A maior diferença de reflectância ocorreu entre oeste-sul, e a menor ocorreu entre nortesul. A maior diferença pode ser notada visualmente na figura 3, na qual o oeste, nessa banda, no geral, manteve-se com maior reflectância que a posição sul.

As maiores diferenças encontradas em alguns pares podem estar relacionadas com a menor ou maior incidência de sol nessas posições, pois a região do visível é influenciada pela quantidade de clorofila das folhas e esta é diretamente afetada incidência de luz sobre a folha.

Para a Banda 2 (700 a $1000 \mathrm{~nm})$, a análise de variância (ANOVA) apresentou diferença significativa entre os valores médios de reflectância entre os tratamentos (Tabela 3). Na tabela 4, observou-se diferença estatística significativa, pelo teste de Tukey HSD, apenas entre os valores médios das reflectâncias no par de posições leste-oeste, o que contrasta com a Banda 1 (400 a $700 \mathrm{~nm}$ ), que obteve diferença significativa entre as reflectâncias médias de todos os pares.

Tabela 3. ANOVA para Banda $2(700 \mathrm{a} 1000 \mathrm{~nm})$, para as orientações norte, sul, leste e oeste do povoamento. Table 3. ANOVA in Band $2(700$ a $1000 \mathrm{~nm})$, for guidelines North, South, East and West of the population.

\begin{tabular}{lccccc}
\hline Fator de variação & GL & SQ & QM & P valor calc. & P valor tab. \\
\hline Posição (árvore) & 3 & 0,0454 & 0,015144 & 3,3264 & $0,01906^{*}$ \\
Erro & 1200 & 5,4631 & 0,004553 & & \\
\hline
\end{tabular}

Tabela 4. Interações ocorridas entre as posições na Banda 2 (700 a $1000 \mathrm{~nm}$ ).

Table 4. Interactions between positions in Band 2 (700 to $1000 \mathrm{~nm}$ ).

\begin{tabular}{lcccc}
\hline Posição & $\begin{array}{c}\text { Diferença } \\
\text { (diff) }\end{array}$ & $\begin{array}{c}\text { Limite inferior } \\
\text { (lwr) }\end{array}$ & $\begin{array}{c}\text { Limite superior } \\
\text { (upr) }\end{array}$ & P adj \\
\hline Leste-Oeste & 0,01689 & 0,00274 & 0,03104 & 0,01167 \\
\hline
\end{tabular}




\section{CONCLUSÕES}

- A reflectância seguiu a mesma tendência para os diferentes tratamentos. A resposta espectral das folhas revelou-se estatisticamente diferente a um nível de $95 \%$ de confiança, ou seja, a posição de coleta das folhas de Eucalyptus grandis varia de acordo com a orientação solar.

- A Banda 1 obteve melhores respostas espectrais em comparação com a Banda 2 do infravermelho próximo (700 a $1000 \mathrm{~nm}$ ), diferenciando a incidência de luz nas posições das folhas obtidas, o que possibilita, dessa forma, a caracterização da absorção da luz solar no visível (400 a $700 \mathrm{~nm})$ em cada posição da árvore.

\section{AGRADECIMENTOS}

À Fundação Estadual de Pesquisa Agropecuária (FEPAGRO), pela doação do material analisado; ao laboratório de sensoriamento remoto da UFSM, pela disponibilização da aparelhagem necessária à realização do trabalho; e ao Programa Nacional de Cooperação Acadêmica (PROCAD).

\section{REFERÊNCIAS}

ABRÃO, P. U. R.; GIANLUPPI, D.; AZOLIN, M. A. D. Levantamento semidetalhado dos solos da estação experimental de silvicultura de Santa Maria. Porto Alegre, 1988. (Publicação IPRNR, n. 21).

ASSOCIAÇÃO BRASILEIRA DE PRODUTORES DE FLORESTAS PLANTADAS (ABRAF). Anuário estatístico da ABRAF 2010 ano base 2009/ ABRAF. - Brasília, 2010. 140p

BRANDELEIRO, C. Espectrorradiometria do visível e infravermelho próximo em povoamento de Eucalyptus grandis W. Hill ex Maiden. 90 f. Tese (Doutorado em Engenharia Florestal) - Universidade Federal de Santa Maria, Santa Maria, 2010.

BRASIL. Ministério da Agricultura. Departamento Nacional de Pesquisa Agropecuária. Divisão pedológica. Levantamento de reconhecimento dos solos do estado do Rio Grande do Sul. Recife, 1973. 413 p. (DNPEA. Boletim técnico, 30).

CLEVERS, J. G. P. W. The application of a weighted infrared-red vegetation index for estimating leaf area index by correcting for soil moisture. Remote Sensing of Environment, New York. v. 29, jul., p. 25 - 37, 1989.

CURRAN, P. J. ; DUNGAN, L. J.; MACLER, A. B.; PLUMMER, E. S. The effect of a red leaf pigment on the relationship between red edge and chlorophyll concentration. Remote Sensing of Environment, New York, v. 35, jan., p. 69 - 76, 1990.

DATT, B. Remote sensing of chlorophyll a, chlorophyll b, chlorophyll a+b, and total carotenoid content in Eucalyptus leaves. Remote Sensing of Environment, New York, v. 1, n. 66, p. 111 - 121, 1998.

EMPRESA BRASILEIRA DE PESQUISA AGROPECUÁRIA (EMBRAPA). Centro Nacional de Pesquisa de Solos. Sistema Brasileiro de Classificação de Solos. Brasília: Embrapa Produção de Informação, 1999. 412 p.

EPIPHANIO, J. C. M.; FORMAGGIO, R. A.; VALERIANO, M. M.; OlIVEIRA; B. J. de. Comportamento espectral de solos do estado de São Paulo. São José dos Campos, 1992.

GATES, D. M.; GATES, H. J.; GATES, J. C.; GATES, V. R. Spectral properties of plants. Applied Optics, v. 4, n. 1, p. 11 - 20, 1965.

GAUSMAN, H. W. Leaf reflectance of near-infrared. Photogrammetric Engineering and Remote Sensing, v. 51, n. 11, p. 1725 - 1734, 1985.

JENSEN, J. R. Sensoriamento remoto do ambiente: uma perspectiva em recursos terrestres. Tradução José Carlos Neves Epiphanio (coord.) [et al]. São José dos Campos: Parêntese, 2009. 598 p. 
MOREIRA, M. A. Fundamentos do sensoriamento remoto e metodologias de aplicação. 3. ed. atual. ampl. Viçosa: UFV, 2007. 320 p. il.

MORENO, J. A. Clima do Rio Grande do Sul. Porto Alegre: Secretaria da Agricultura, 1961. 46 p.

PEÑUELAS, J.; FILELLA, I. Visible and near-infrared reflectance techniques for diagnosing plant physiological status. Trends in Plant Science, v. 3, abril, p. 151 - 156, 1998.

PONZONI, F. J. Comportamento spectral da vegetação. In: MENESES, P. R.; MADEIRA NETO, J. S. Sensoriamento remoto: reflectância de alvos naturais. Brasília, DF: UNB Planaltina Embrapa Cerrados, 2001. $262 \mathrm{p}$.

PONZONI, F. J.; SHIMABUKURO, Y. E. Sensoriamento remoto no estudo da vegetação. São José dos Campos: Parêntese, 2010. 127 p.

PREVEDELlO, J. Preparo do solo e crescimento inicial de Eucalyptus grandis Hill ex Maiden em Argissolo. Dissertação (Mestrado em Engenharia Florestal) - Universidade Federal de Santa Maria, Santa Maria, 2008.

SCHEPERS, J. S.; BLACKMER, M. T.; WILHELM, W.; RESENDE, M. Transmittance and reflectance measurements of corn leaves from plants with different nitrogen and water supply. J. Plant Physiological, v. 148, p. 523 - 529, 1996.

STRECK, E. V.; KÄMPF, N.; DALMOLIN, R. S. D.; KLAMT, E.; NASCIMENTO, P. C.; SCHNEIDER, P. Solos do Rio Grande do Sul. Porto Alegre: EMATER/RS, UFRGS, 2002. 107 p.

THENKABAIL, P. S.; SMITH, R. B.; De-PAUW, E. Hyperspectral vegetation indices for determining agricultural crop characteristics. Remote Sensing of Environment, v. 71, p. 158 - 182, 2000.

TRIGG, S.; FRASSE, S. Characterizing the spectral-temporal response of burned savannah using in situ spectroradiometry and infrared thermometry. International Journal of Remote Sensing, v. 21, 2000. 